\title{
Fast Sphere Detection Based on Polytope Method Using One-Dimensional Histogram
}

\author{
Shota Nakashima ${ }^{\mathrm{a}, *}$, Hiroyuki Nakamoto ${ }^{\mathrm{b}}$, Shenglin $\mathrm{Mu}^{\mathrm{c}}$, \\ Yuhki Kitazono ${ }^{\mathrm{d}}$, Huimin $\mathrm{Lu}^{\mathrm{e}}$ and Kanya Tanaka ${ }^{\mathrm{a}}$ \\ ${ }^{a}$ Graduate School of Science and Engineering, Yamaguchi University, Ube 755-8611, Japan \\ ${ }^{\mathrm{b}}$ Department of Computer Science, Nagoya Institute of Technology, Nagoya 466-8555, Japan \\ ${ }^{\mathrm{c}}$ Department of Electronic Control Engineering, Hiroshima National College of Maritime Technology, \\ Toyota-gun 725-0231, Japan \\ ${ }^{\mathrm{d}}$ Department of Electronic and Control Engineering, Kitakyushu National College of Technology, \\ Kitakyushu 802-0985, Japan \\ ${ }^{\mathrm{e}}$ Department of Electrical Engineering and Electronics, Kyushu Institute of Technology, \\ 1-1 Sensui-cho, 804-8550, Japan \\ *Corresponding Author: s-naka@yamaguchi-u.ac.jp
}

\begin{abstract}
We propose a method for fast extraction of sphere. Basically, main algorithms of the extraction in 3D figure are the same as $2 \mathrm{D}$ method we reported in previous research. The proposed method utilizes the one-dimensional histogram as search space, and the polytope method which is one of the minimization algorithms for search parameters in target figure. The histogram has two characteristics: (a) The distribution of the histogram changes if the parameters of representing the sphere changes. (b) The value of highest frequency of histogram becomes maximum if the best parameters are obtained. Therefore, the maximum value of highest frequency of histogram is searched to obtain the best parameters of the sphere by using the polytope method. By using the polytope method, the proposed method can extract the sphere from 3D vertex data without a large memory space or long processing time.
\end{abstract}

Keywords: 3D pattern matching, polytope method, figure detection.

\section{Introduction}

In factory automation, recognition of object and detection of position is necessary in assembly ${ }^{(1)}$. Human interface devices which recognize humans and things to input gaming consoles are applied ${ }^{(2)}$. Recognition of objects or humans is needed in field of welfare. In these fields, 3D pattern matching is an important technique. We have proposed previously a fast extracting method for 2D figure with lines, circles and arbitrary shapes using small memory space $^{(3-5)}$. Furthermore, the 2D extraction method was developed to extract a sphere ${ }^{(6)}$. In this paper, it is the first time to implement the proposed method in sphere extraction. Different from conventional silhouette method ${ }^{(7)}$, the proposed method is designed to extract the sphere from $3 \mathrm{D}$ vertex data which is gotten by using stereo camera ${ }^{(8)}$ or 3D laser scanner ${ }^{(9)}$. According to the $3 \mathrm{D}$ extraction in previous research $^{(10)}$, we employ other target figures and their evaluation value for extraction in this paper. Based on the $2 \mathrm{D}$ method we reported in previous research, we describe our proposed method as 2D method's extension to recognize sphere, using the polytope method and one-dimensional histogram, from target figures in this paper.

\section{Sphere Extraction}

The proposed method utilizes the polytope method and one-dimensional histogram. An application of the polytope method to extract figures and a procedure of extraction of figures using one-dimensional histogram are introduced in this section.

\subsection{Approach}

In this study, one-dimensional histogram is generated 


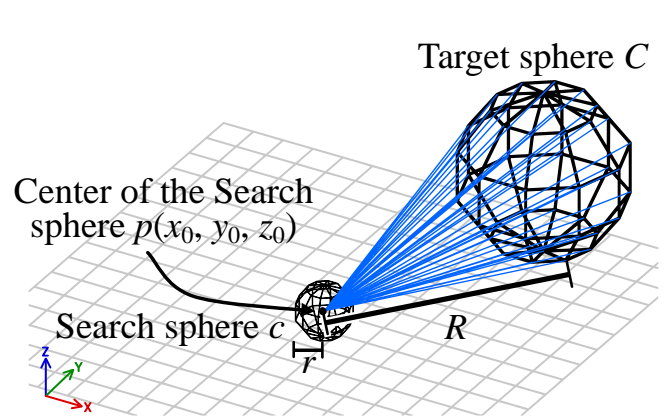

(a) Initial position

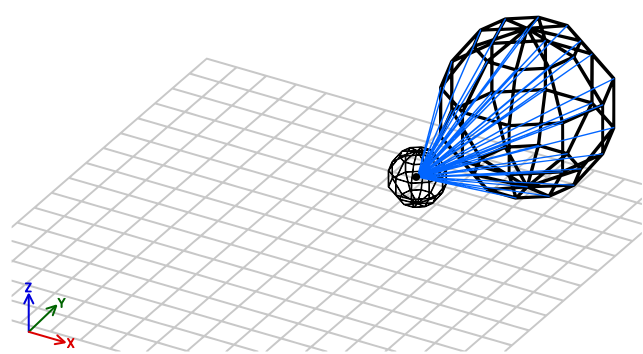

(b) Searching

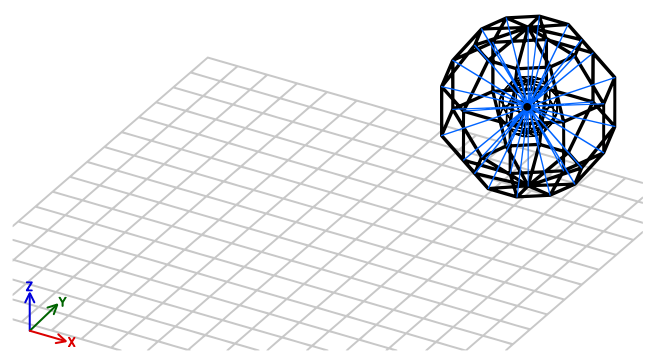

(c) Search completed

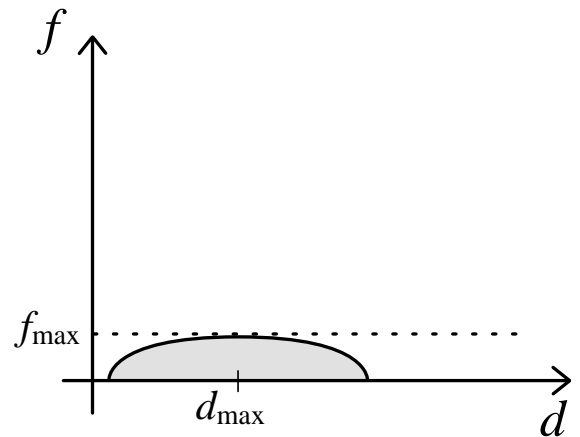

(d) One-dimensional histogram of (a)

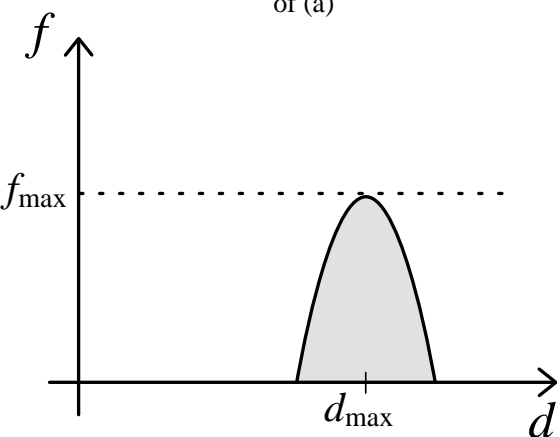

(e) One-dimensional histogram of (b)

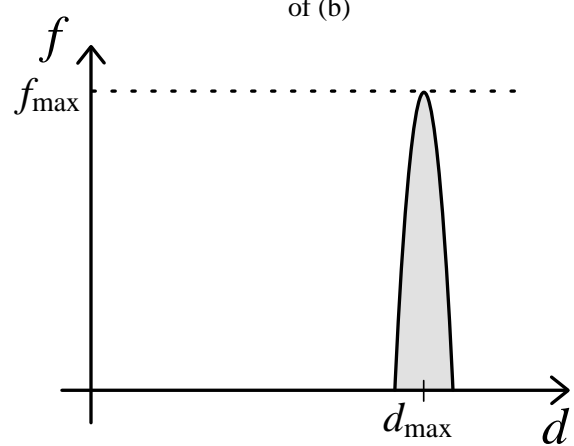

(f) One-dimensional histogram of (c)

Fig. 1. Vertex of search for extraction of 3D sphere

from 3D vertex data. The histogram has two characteristics. (a) The distribution of the histogram changes if the parameters of representing the sphere changes. (b) The value of highest frequency of histogram becomes maximum if the best parameters are obtained (See the section called "Extraction Method of sphere" for details). By using the polytope method, the maximum value of highest frequency of histogram is applied to obtain the best parameters of a sphere.

The polytope method has three characteristics:

(1) Since it can get a minimum value without derived function different from the Newton's $\operatorname{method}^{(11)}$, the concept can be used for searching in histogram.

(2) Light estimation in program.

(3) For the use, the "initial values" must be set because this method is available for only a single-peak function. If these are not suitable, the optimum value may not be obtained.

For more details, see Ref. (12) and (13).

\subsection{Extraction Method of Sphere}

Extraction procedure of sphere using one-dimensional histogram is introduced in this section. A search sphere $c$ is defined for the extraction of target sphere $C$ as shown in Fig.1a. To represent search sphere $c$ as parameters $p\left(x_{0}, y_{0}\right.$, $z_{0}$ ), center coordinates of search sphere $c$, is defined as shown in Fig.1a. The distance between center of search sphere $c, p\left(x_{0}, y_{0}, z_{0}\right)$, and a point on target sphere $C$ is set as $R$. The distance of search sphere $c$ to the direction of a point on $c$ is $r$, and let $R / r$ be the distance ratio $d$. The value of $d$ is calculated for all vertexes on target sphere $C$ to make 
one-dimensional histogram with $x$ axis $d$ and $y$ axis frequency $f$. The value of highest frequency is defined as $f_{\text {max }}$.

If the parameters of search sphere $c$ which are represented by $p\left(x_{0}, y_{0}, z_{0}\right)$ are much different from those of target sphere $C$, the deviation of distance ratio $d$ is large. Thus, $d$ is voted on wide part of one-dimensional histogram. As a result, the distribution of the histogram is gentle. In consequence, the value of highest frequency $f_{\max }$ is low as

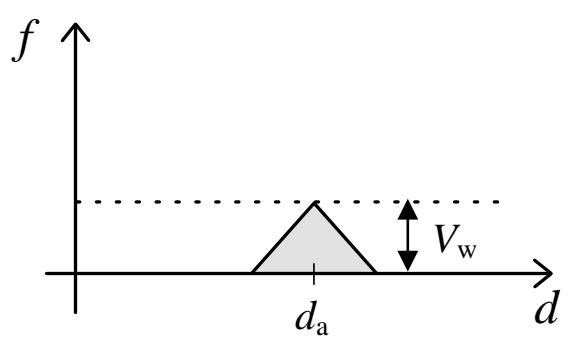

Fig. 2. Weight of voting $V_{\mathrm{w}}$

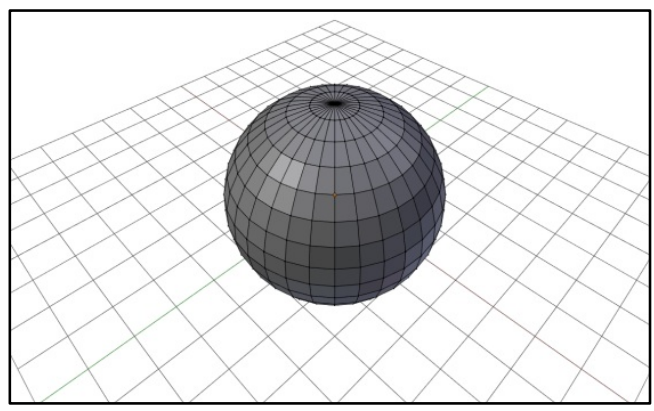

(a) Normal sphere shown in Fig.1d. Here, we define the value of $d$ at the position of highest frequency $f_{\max }$ as $d_{\max }$.

According to the parameters of search sphere $c$ approach those of target sphere $C$ (Fig.1a and Fig.1b), the

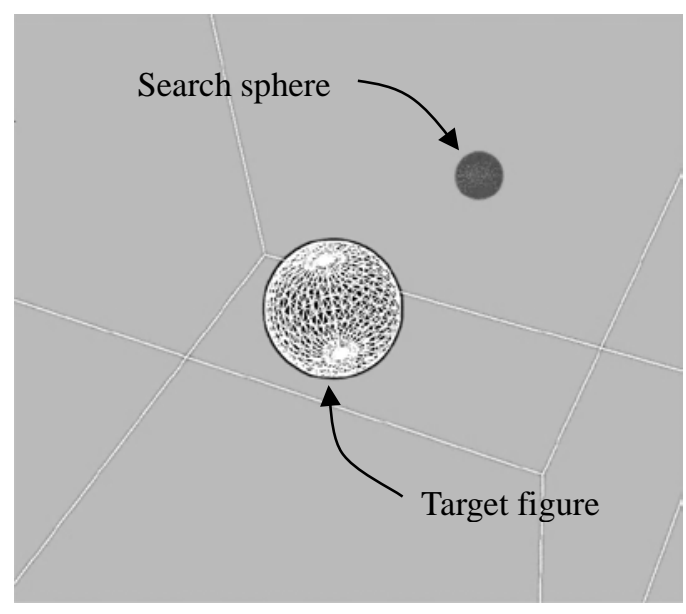

Fig. 3. Initial setting of search sphere and target figure in searching space

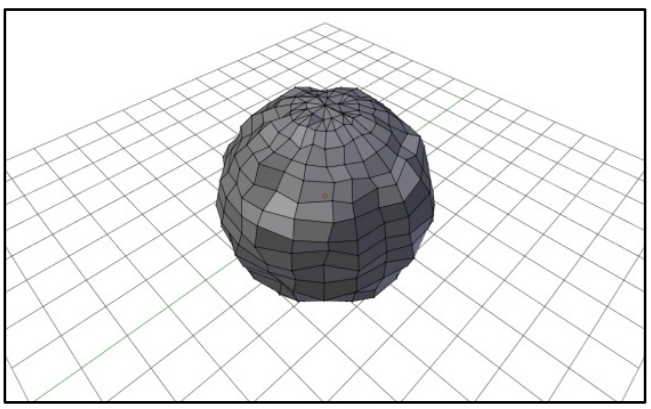

(b) Odd sphere

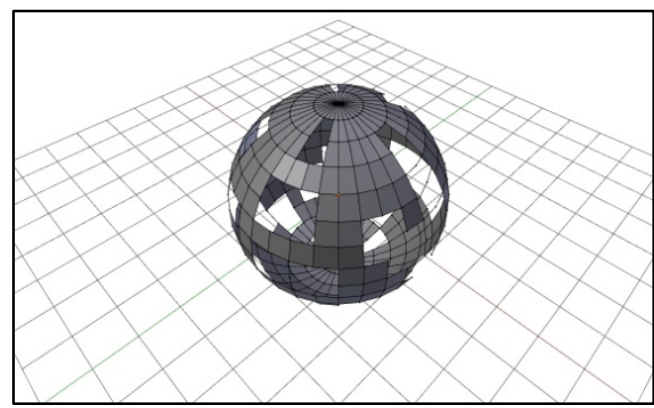

(c) Chipped sphere

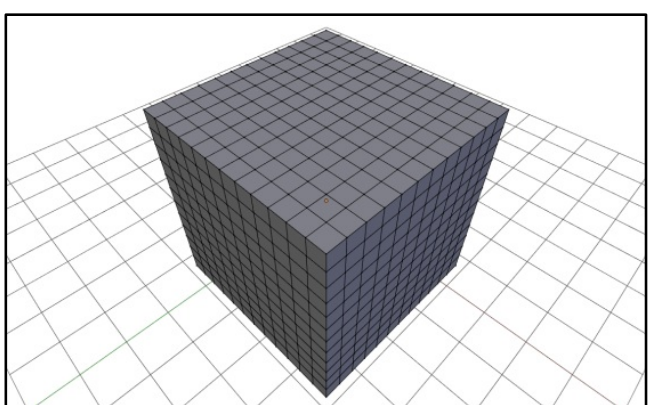

(d) Cube

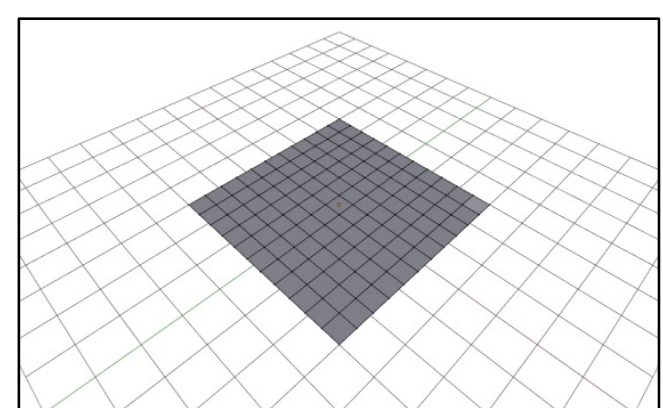

(e) Plane

Fig. 4. Vertex data of target figures 
Table.1 Extraction results

\begin{tabular}{ccccccc} 
Target figure & \multicolumn{9}{c}{ Parameters } & Evaluation & $\begin{array}{c}\text { Proccessing } \\
\text { value } E\end{array}$ & time [s] \\
\cline { 2 - 5 } & $x$ & $y$ & $z$ & $R_{\max }$ & 0.077 & 220 \\
\hline Normal sphere & 0.01 & -0.05 & -0.01 & 3.00 & 0.07 & 230 \\
Odd sphere & 0.01 & -0.05 & -0.01 & 3.00 & 0.161 & 160 \\
Chipped sphere & -0.03 & -0.03 & -0.10 & 2.90 & 0.108 & 280 \\
Cube & 0.06 & 0.05 & 0.06 & 3.80 & 0.393 & 160 \\
Plane & 1.07 & 1.01 & -1.01 & 2.20 & 0.848 & \\
\hline
\end{tabular}

deviation of $d$ becomes small. Thus, $d$ is voted on part of one-dimensional histogram. Then, the value of highest frequency $f_{\max }$ becomes high as shown in Fig1d and Fig1e. If the parameters of search sphere $c$ agree with those of target sphere $C$ completely as shown in Fig.1c, all of distance ratio $d$ is same value. Thus, distance ratio $d$ is voted on limited part of one-dimensional histogram. There for, the value of highest frequency $f_{\max }$ is the highest as shown in Fig.1f. The scale of search sphere $c$ is defined as $s$. In this study, $s$ is set to 1 . At the last case, the scale $S$ of target sphere $C$ is obtained as $s \times d_{\max }$. In this way, the target sphere $C$ is extracted if the value of highest frequency $f_{\max }$ of histogram becomes maximum. Thus, we define the following evaluation function $E$ to evaluate the histogram.

$$
E=1-\frac{f_{\max }}{4 \pi d_{\text {max }}^{2} \times N_{v} \times V_{w}}
$$

where, $N_{\mathrm{v}}$ is the number of the vertexes per area. Symbol $V_{\text {w }}$ means a weight which is used when $d$ is voted to one-dimensional histogram. The weight has a distribution. The example is shown in Fig.2. If the parameters of search sphere $c$ are the same with those of target sphere $C, f_{\max }$ is highest. Then, the value of function $E$ becomes the lowest. $p\left(x_{0}, y_{0}, z_{0}\right)$ of target sphere $C$ is searched, so that the function $E$ is lowest, by using the polytope method. As shown in Fig. 3, the center of target figure is set at $(0,0,0)$ and the search sphere is at $(5,5,5)$.

\section{Experiment}

3D vertex data of target figures are applied in experiments to detect the normal sphere (Fig.4 (a)), odd sphere (Fig.4 (b)), chipped sphere (Fig.4 (c)), cube (Fig.4 (d)) and plane (Fig.4 (e)). The number of vertexes are, normal sphere (Fig.4 (a)) 482, odd sphere (Fig.4 (b)) 482, chipped sphere (Fig.4 (c)) 455, cube (Fig.4 (d)) 866, plane (Fig.4 (e)) 144, respectively. The number of the vertexes per area $N_{\mathrm{v}}$ was set to 4.26 . In each experiment, the search

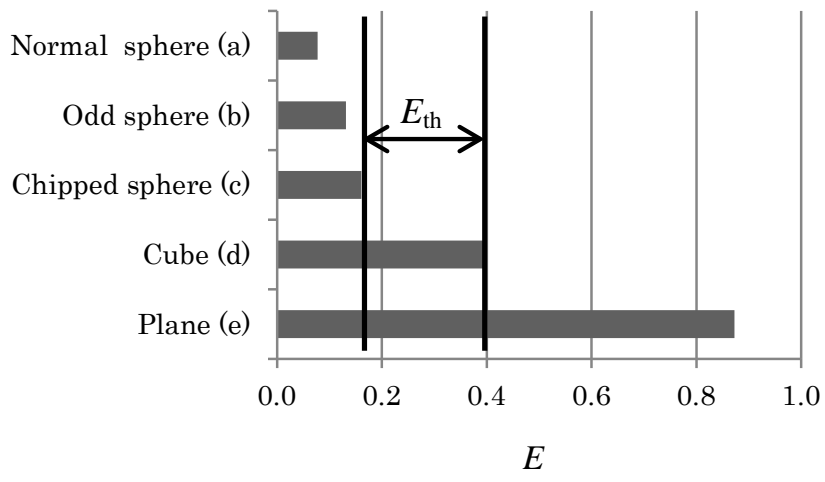

Fig. 5. Results of evaluation value E.

sphere is set to $p\left(x_{0}, y_{0}, z_{0}\right)=(5,5,5), r=1$, and the each position of target figures is set to $p(x, y, z)=(0,0,0) . V_{\mathrm{w}}$, weight used when voting to one-dimensional histogram, is set to 10. In the experiments, we used a personal computer (CPU: E-350 1.6[GHz] (AMD, Inc.), OS: Momonga Linux 7 x86_64). The range of search space was set to all $x-y-z$ axis as \pm 15 distance from $(0,0,0)$. Let the distance ratio $d$ of point on figure $C$ be $d_{\mathrm{a}}$. First, the value of $V_{\mathrm{w}}$ is voted at the position of $d_{\mathrm{a}}$ in one-dimensional histogram. According to that, as the distance ratio $d$ goes away from $d_{\mathrm{a}}$, the value will reduce one by one. $d$ was calculated for all vertexes by the method introduced in Sect.2.2.

As we see in the experimental results, value of $E$ and parameters after the process are shown in Table 3 . The comparison of $E$ in searching of each shape is shown in Fig.5. Obvious difference of $E$ of different shapes can be seen in Fig. 5. According to the experiments, by setting the value of $E_{\text {th }}$ between 0.17 and 0.39 , we can separate sphere or similar sphere from other shapes.

\section{Conclusions}

This paper reports our method of extracting sphere by using one-dimensional histogram. Owing to the simplicity of the polytope method and the one dimension histogram which is light to apply are employed, the proposed method 
only expends very small memory space and requires short processing time. The algorithm is robust and against noise. Also, it can separate sphere and other shapes. In addition, proposed method can be developed to extraction of arbitrary 3D shapes.

\section{Acknowledgment}

This work was supported by JSPS KAKENHI Grant Number: 24700198.

\section{References}

(1) Manabu Hashimoto and Kazuhiko Sumi : “3-D Object Recognition Based on Integration of Range Image and Gray-scale Image”, Proceedings of The British Machine Vision Conference 2001, pp. 253-262, 2001

(2) Jamie Shotton, Andrew Fitzgibbon, Mat Cook, Toby sharp, Mark finocchio, Richard Moore, Alex Kipman, and Andrew Blake : "Real-Time Human Pose Recognition in Parts from Single Depth Images", Proceedings of Computer Vision and Pattern Recognition 2011, 2011

(3) Hiroshi Kimura, Tomoyuki Horiuchi, and Katsushi Ikeuchi : "Human Robot Cooperation for Mechanical Assembly using Cooperative Vision System”, 2nd Int. Workshop on Cooperative Distributed Vision, pp.213-228, 1998

(4) Yue Liu, Yuhki Kitazono, Shota Nakashima, Lifeng Zhang, and Seiichi Serikawa : "Proposal of Driver Symbol Mark Detection using One-Dimensional Histogram Figure Extraction Method”, Applied Mechanics and Materials, Vol.36, pp.376-380, 2010

(5) Shota Nakashima, Makoto Miyauchi, and Seiichi Serikawa : "Proposal of a method to extract of arbitrary figures using one-dimensional histogram”, Journal of Advanced Computational Intelligence and Intelligent Informatics, Vol.13, No.4, pp.380-385, 2009

(6) Shota Nakashima and Seiichi Serikawa : "Proposal of a method to extract straight line and circle using one-dimensional histogram”, Journal of Artificial Life and Robotics, Vol.13, No.1, pp.306-310, 2008

(7) Yoshihiro Okada : "3D Model Matching Based On Silhouette Image Matching”, Proceedings of Circuits/Systems, Computers and Communications 2002, WSEAS Press, pp. 380-385, 2002

(8) Louis-Philippe Morency, Ali Rahimi, and Trevor Darrell : "Fast 3D Model Acquisition from Stereo
Images”, Proceedings of 3D Processing • Visualization - Transmission, pp. 172-176, 2002

(9) Lijing $\mathrm{Bu}$ and Zhengpeng Zhang : “Application Of Point Clouds from Terrestrial 3D Laser Scanner for Deformation Measurements”, Proceedings of International Society for Photogrammetry and Remote Sensing, Vol. XXXVII, Part B5, pp.545-548, 2008

(10) Andrew Schworer and Peter Hovey : "Newton-Raphson Versus Fisher Scoring Algorithms in Calculating Maximum Likelihood Estimates”, Proceedings of Undergraduate Mathematics Day, 2004

(11) Shota Nakashima, Hiroyuki Nakamoto, Yuhki Kitazono, Lifeng Zhang and Seiichi Serikawa : “A Method for sphere Detection Using One-Dimensional Histogram and Polytope Method”, Applied Mechanics and Materials, Vol.103, pp.628-632, 2011

(12) Haruhiko Okumura : “An algorithm encyclopedia using C”, Gijutsu-Hyohron Co.,p.262, 2006

(13) W. H. Press, B. P. Flannery, S. A. Teulolsky, and W. T. Vetterling : "Numerical Recipes-The Art of Scientific Computing”, Cambridge University Press, pp. 289-293, 1987 